\title{
READY PLAYER ONE - LERNJOURNALE/LERNTAGEBÜCHER IN DER AUSBILDUNG VON DAF/DAZ-LEHRENDEN. EIN PRAXISBEITRAG FÜR ERFAHRUNGEN UND VORSCHLÄGE FÜR NEUE EXPERIMENTE
}

\author{
ANNA MAJOROSI PHD, Hochschulprofessorin \\ KODOLÁNYI JÁNOS Egyetem \\ majorosianna@kodolanyi.hu
}

DOI 10.47273/AP.2020.20.40-50

\begin{abstract}
ABSTRAKT
In meinem Praxisbeitrag geht es um den Einsatz von Lerntagebüchern/Lernjournalen im universitären Kontext, in der Ausbildung von DaF/DaZ-Lehrenden. Es werden vor allem konkrete Erfahrungen und Vorschläge aus der Praxis beschrieben, die die Leserinnen und Leser (Hochschulangehörige) zum Experimentieren einladen möchten.
\end{abstract}

Schlüsselwörter: Lernjournale, Lerntagebücher, Reflexivität, DaF/DaZ-Studierende, Stufen der Reflexivität

\begin{abstract}
In my article I am presenting the use of learning diaries in university education with special regards to German Language undergraduates. I am describing concrete, practical experiences and giving suggestions which, hopefully will make lecturers willing to use them in their professional work.
\end{abstract}

Keywords: learning diary, learning journal, reflexivity, student-teacher of German language, levels of reflexivity

\section{Gründe für den Einsatz von Lernjournalen}

Seit einem Jahrzehnt beschäftige ich mich mit der Problematik des reflexiven Denkens in diversen Bildungskontexten und dessen didaktischen Instrumenten wie Portfolios, Lernjournale/Lerntagebücher oder Feedbacks. Ich leite die Studierenden an, 
Lernjournale/Lerntagebücher in diversen Kontexten in Österreich und Ungarn zu schreiben: in der Ausbildung von künftigen DaF/DaZ-Unterrichtenden, in der Fortbildung von DaF/DaZLehrenden, in der Weiterbildung von Schulleiterinnen und Schulleitern und in der Berufsbildung. In diesem Beitrag fokussiere ich auf die Ausbildung und Fortbildung von DaF/DaZ-Lehrenden; die Beispiele stammen aus meiner Lehrpraxis an der Universität Wien. Ich bin nicht die Einzige, die mit dem Einsatz von Lernjournalen/Lerntagebüchern experimentiert, aber die Praxisberichte aus Hochschulinstitutionen in diesem Bereich sind relativ selten. Aus diesem Grund möchte ich die Argumente, die für das Verfassen von Lernjournalen/Lerntagebüchern sprechen, nur stichwortartig auflisten. In dem Beitrag möchte ich einerseits konkrete Beispiele zeigen, andererseits Vorschläge für den Einsatz von Lernjournalen/Lerntagebüchern im universitären Bereich skizzieren, um den Kolleginnen und Kollegen bei demselben eine mögliche Hilfe zu leisten. Dieser Beitrag richtet sich in erster Linie an FachdidaktikerInnen und an Unterrichtende an diversen Hochschulinstitutionen, und die Art und Weise des Beitrags entspricht der schweizerischen hochschuldidaktischen Texten.

Gründe für die bewusste Förderung der Reflexionsfähigkeit durch den Einsatz von Lernjournalen/Lerntagebüchern:

- gesellschaftliche Veränderungen (Veränderung der Beziehungen, mangelnde Stabilität, heterogene Umwelt, Individualisierung)

- das sogenannte Lebensprojekt erfordert den professionellen inneren Dialogpartner

- Selbstreflexion kann nur inhaltsbezogen entwickelt werden

- der Perspektivenwechsel (Lernende - Lehrende) wird ermöglicht

- Transfer zum (künftigen) Unterricht wird schriftlich festgehalten

- authentische Schreibmöglichkeit

- Lerntagebücher sind Umsetzungen der konstruktivistischen Lerntheorien

- die durch Asymmetrie oder Hierarchie gekennzeichnete Beziehung zwischen Unterrichtenden und Studierenden wird aufgehoben

- die Selbstwahrnehmung und die sich daraus ableitende Selbstwirksamkeit der Individuen werden mit diesem Instrument gefördert

- die vier Zustandsvariablen der Verhaltenssteuerung (die physische, die emotionale, die kognitive und die soziale) werden bewusstgemacht

- das Selbstbewusstsein wird gestärkt, was zur Entwicklung des Ich-s (des Selbstmodells) beiträgt 
- sowohl die Reflexion während der Handlung, als auch die Reflexion nach der Handlung (vgl. Schön 1983) wird durch das Lernjournalschreiben ermöglicht, das zur Professionalisierung als Lehrperson führt

\section{Ein Beispiel aus der Praxis}

In der Ausbildung von künftigen Lehrenden gibt es genügend Zeit, das Verfassen von Lernjournalen/Lerntagebüchern in zwei Schritten aufzubauen. Im ersten Schritt wird der Fokus auf die Beobachtung, auf die Reflexion des Lerngegenstandes, der Lernhandlung, evtl. der Lernhaltung, der Gruppenprozesse und des Transfers gelegt. An der Universität Wien habe ich die Aufgabe folgenderweise eingeführt (Auszug aus der Lehrveranstaltungsbeschreibung, um den genauen Kontext der Lernjournale und der Wahlmöglichkeiten vorzuzeigen):

\section{„Aufgaben und Übungen: Förderung der allgemeinen Kompetenzen}

im DaF/DaZ-Unterricht"

\section{Ziele der Lehrveranstaltung:}

die StudentInnen sind im Stande, die Problematik der Förderung der allgemeinen Kompetenzen zu reflektieren

$\square \quad$ die StudentInnen kennen mind. 4 Konzepte der Erwachsenenbildung

$\square$ die StudentInnen können Aufgaben zur Förderung der allgemeinen Kompetenzen in ihrem Unterricht einsetzen

$\square$ die StudentInnen können ihre soft skills einschätzen und die Ergebnisse in ihre Überlegungen/Unterrichtsplanung einbeziehen

Inhalte: Grundbegriffe, Prinzipien des Sprachunterrichts und die allgemeinen Kompetenzen, Unterschied zwischen Aufgaben und Übungen, zielorientierte Planung, allgemeine Kompetenzen nach dem GER, persönliche und soziale Kompetenzen, Lernen Erwachsener, Lernen Jugendlicher, Konzepte der Erwachsenenbildung, ethischmoralische Fragen des Themas, Hierarchisierung der soft skills, Reflexivität, lernpsychologische online Tests, Aufgaben und Materialien zur Förderung der allgemeinen Kompetenzen, Lehrwerkanalyse

Leistungsnachweis: für Alle: A) selbständige Bearbeitung und die Präsentation der wichtigsten Punkte eines freigewählten Fachtextes zu einem der obigen Inhalte bis zum zweiten Block +

B) von den folgenden Möglichkeiten sollen mind. zwei geleistet werden, eine davon schriftlich: Lernjournal (vgl. Leitfragen) über die ganze LV, Hausarbeit, Präsentation von einer selbst erarbeiten Fragestellung, Vorstellung ausgewählter und erprobter Aufgaben zur Förderung der allgemeinen Kompetenzen in einer Gruppe, Vorstellung kreativer Aufgaben mit didaktischer Begründung, Vorstellung einer eigenen Mini-Forschung zum Thema Förderung der allgemeinen Kompetenzen, Vorstellung eines eigenen Films oder Hörspiels zum Thema (einige Arbeiten können in Partnerarbeit oder in einem kleinen Team auch gemacht werden)

- es können aber auch weitere Vorschläge besprochen werden

Methoden: In der Lehrveranstaltung wird mit erwachsenengerechten Methoden gearbeitet, die im Sprachunterricht auch später verwendet werden können.

\section{Lernjournal}

Das Lernjournal ist ein Instrument, das dabei helfen soll, das im Lehrgang Gehörte, Beobachtete und Gelernte nochmals zu reflektieren und daraus Konsequenzen für den eigenen Unterricht abzuleiten. 
Im Französischen bedeutet „réfléchir“ nachdenken und auch im Lernjournal geht es in erster Linie darum, zu überlegen und nachzudenken, was meinen Lernprozess in den diversen Dimensionen beeinflusst, wo es (Handlungs-)Blockaden gibt und wie bzw. wann Lernen (am) erfolgreich(sten) sein kann.

\section{Die Ziele der Lernjournalführung sind:}

- $\quad$ über die Inhalte, Methoden und Gruppenprozesse zu reflektieren

- $\quad$ die eigenen Ziele und Lernfortschritte bewusst zu machen

- $\quad$ Erfahrungen mit der Führung des Lernjournals zu sammeln und dadurch zu erkennen, was Selbstreflexion bedeutet

- den Transfer in den eigenen Unterricht herzustellen.

Achtung: Das Lernjournal ist ein halböffentliches Dokument!

Beim Lernjournalschreiben ist es wichtig, die Reflexion des Beobachteten und die Relevanz für das eigene Tun vor Augen zu haben.

\begin{tabular}{|c|c|c|}
\hline Was? & Wie? & Transfer in den eigenen Unterricht \\
\hline $\begin{array}{l}\text { Was habe ich in diesem } \\
\text { Block gelernt? (fachlich, } \\
\text { methodisch, sozial) }\end{array}$ & $\begin{array}{l}\text { Wie habe ich die Arbeitsformen erlebt? } \\
\text { Was beschäftigt mich im Zusammenhang } \\
\text { mit dem bearbeiteten Inhalt? Mit welchen } \\
\text { Inhalten habe ich (noch) Schwierigkeiten? } \\
\text { Wo gibt es Stolpersteine, wo verstehe ich } \\
\text { etwas nicht? Was hat mir besonders gut } \\
\text { gefallen und warum? } \\
\text { Was haben mir die Gruppenarbeiten / } \\
\text { Partnerarbeiten gebracht? } \\
\text { Was ist schwierig und was ist besonders } \\
\text { angenehm in der Gruppe? }\end{array}$ & $\begin{array}{l}\text { Was bringt mir das Gelernte (fachlich, } \\
\text { methodisch, sozial) für meinen (künftigen) } \\
\text { Unterricht? } \\
\text { Wie kann ich das Gelernte in meinem } \\
\text { (künftigen) Unterricht konkret umsetzen? }\end{array}$ \\
\hline
\end{tabular}

Form und Länge des Lernjournals hängen vom Verfasser/von der Verfasserin ab. Wichtig: Machen Sie sich während der LV Notizen für das Lernjournal und schreiben Sie Ihr LS möglichst bald (innerhalb einer Woche) nach dem aktuellen Block.““

Wie aus der obigen Beschreibung entnommen werden kann, lege ich besonders viel Wert auf die Förderung der Entscheidungsfähigkeit der Studierenden, und ermögliche ihnen, das Verfassen von Lernjournalen frei zu wählen. Nach meiner Erfahrung wählen ca. 75\%-85\% der Studierenden diese Art des Leistungsnachweises. Ihre Motivation für ihre Entscheidung, die sie in den Lernjournalen mitgeteilt haben, ist unterschiedlich: Einige haben davon noch nie gehört und möchten es ausprobieren, andere denken, dass es bedeutend einfacher sein kann, einen persönlichen Text als einen wissenschaftlichen Text zum Thema zu schreiben, und etliche sehen in einem Lernjournal ein Instrument für die Bewusstmachung der eigenen Lernprozesse. 
Am Anfang werden vor allem bezüglich der Länge und der Textsorte Unsicherheiten signalisiert. Ich versuche die Studierenden zu ermutigen, ihren eigenen Weg zu finden, demgemäß schlage ich ihnen vor, sowohl die tabellarische Form als auch den selbststrukturierten und den völlig freien Text in einer Aufgabe zu variieren. Nur ca. $15 \%$ der Studierenden wechselt die Form innerhalb des Lernjournals, die meisten bleiben bei ihrer ersten Entscheidung. Es gibt auch Studierende, die offenbar Angst vor dem leeren Blatt haben, und so die tabellarische Form wählen; unter ihnen finden wir zahlreiche künftige LehrerInnen, die nicht merken, dass der eigene Text die Tabelle sprengt. Das längste Lernjournal, das ich an der Universität Wien nach drei Wochenendblöcken bekommen habe, war ein Text von 72 Seiten. Die durchschnittliche Länge für diese Aufgabe beträgt 12-15 Seiten.

Im zweiten Schritt, in höheren Semestern oder in Gruppen mit besonders ausgeprägter Reflexionskompetenz oder bei auffällig problematischen Lerngruppen, empfiehlt es sich, eine andere Struktur vorzugeben, basierend auf den Stufen bzw. den Zyklen der Reflexivität von Gibbs (1988), weil eine reflexive Haltung die kontinuierliche Selbstbeobachtung und Auseinandersetzung mit sich selbst erfordert. Diese Strukturierung kann Lehramtsstudierende sehr gut auf die diversen Hospitationen, Praktika und Feedbacks vorbereiten:

Stufe 1: Beschreibung der Situationen aufgrund der Beobachtungen (ohne Bewertung)

Stufe 2: Formulierung der Gefühle, die in der Situation erlebt wurden

Stufe 3: Bewertung der Situation aus der eigenen Perspektive (was ist gut und was weniger gut gelungen)

Stufe 4: Kritische, möglichst objektive Analyse der Situation (Standortwechsel)

Stufe 5: Formulierung von Konklusionen, d. h. was hätte man anders machen können/sollen

Stufe 6: Erarbeitung eines Planes für ähnliche Situationen (Transfer und Zukunftsrelevanz)

\section{Einstiegsmöglichkeit durch einen literarischen Text}

Bei den Lernjournalen/Lerntagebüchern haben nicht nur die Lernenden Verpflichtungen, sondern auch die Unterrichtenden. Ich plädiere dafür, dass die Unterrichtenden, wenn Sie ein Lernjournal/Lerntagebuch verlangen, den VerfasserInnen eine kurze (4-5 Sätze) schriftliche Rückmeldung geben. Wenn sie sich dafür auch so viel bzw. wenig Zeit nicht nehmen können, sollten sie auf die Forderung eines Lernjournals/Lerntagebuchs lieber verzichten. 
Vor zwei Jahren wurde ich auf den Film von Steven Spielberg, Ready Player One, aufmerksam, aber wie die meisten meiner Generation ging ich nicht ins Kino, sondern griff sofort nach dem Buch, auf dem er basiert (deutsche Ausgabe 2012). Das Buch, das laut der Filmkritik des ZeitOnline Magazin vom 27.03. 2018 von seinen Fans als Popkultur-Bibel verehrt wird, gehört m. E. zur getarnten Jugendliteratur, darunter zur Jungenliteratur. Leider liest das Zielpublikum des Romans immer weniger (der Roman erschien erst 2018 in ungarischer Übersetzung, so bestand kaum die Gefahr, dass das Buch vor dem Film gelesen wird). Das Buch, das im Jahre 2045 spielt, verarbeitet die Popkultur und die Geschichte der zweiten Hälfte des 20. Jahrhunderts, die ja fast ausschließlich für die Generation 50+ richtig zugänglich bzw. begreifbar ist. Was hat nun aber ein Science Fiction- Jugendbuch mit dem Thema Reflexivität zu tun? Am einfachsten kann man die Frage durch ein Beispiel beantworten, das die Reflexionen des jungen Romanhelden über die Schule zeigt und der als quasi Lernjournaleintrag gelesen werden kann.

Dies kann gleichwohl als Impuls zum Lernjournalschreiben in den Lehrveranstaltungen verwendet werden:

„Unsere Lehrerin, Ms Rank, stand vor der Klasse und konjugierte bedächtig lateinische Verben. Sobald sie ein Wort aussprach - zuerst auf Englisch, dann auf Lateinisch -, tauchte es automatisch auf der Tafel hinter ihr auf. Wenn wir uns mit der Konjugation von Verben abplagten, musste ich jedes Mal an den Text eines alten Liedes aus Schoolhouse Rock! denken: ,To run, to go, to get, to give. Verb! You're what's happening!' Ich summte die Melodie leise vor mich hin, während Ms Rank anfing, das Verb ,lernen`zu konjugieren. ,Lernen. Discere‘, sagte sie. ,Nun, das solltet ihr euch leicht merken können, denn es ist mit dem Wort Disziplin verwandt. Zum Lernen braucht man Disziplin.' Als ich hörte, wie sie das Wort ,lernen“ wiederholte, fiel mir wieder der Limerick ein: Noch viel muss er lernen, noch viel muss er schauen, muss auf sein gewonnenes Wissen bauen, bis ihm ein Platz auf der Liste wird zuerkannt. Als Nächstes verwendete Ms Rank das Verb in einem Satz: ,Wir gehen zur Schule, um zu lernen“, sagte sie. „Ad discendium in scholam imus.“ Tja, und da ging mir plötzlich ein Licht auf. Ein ganzer Kronleuchter. Ich schaute mich im Klassenzimmer um. ..." (Cline, 2012, S. 104)“

Ich hoffe, es ist mir gelungen, das Interesse der Leserinnen und der Leser zu wecken, und sie zum Experimentieren mit Lernjournalen/Lerntagebüchern einzuladen.

\section{Lernjournalauszüge von Studierenden}

Am Ende des Beitrages möchte ich drei Beispiele [unverändert] aus Lernjournalen von Studierenden zeigen, um die Sinnhaftigkeit und die Schwierigkeit der Aufgabe zu demonstrieren. Ich glaube, dass die drei Texte für sich sprechen. Die Texte stammen aus dem ersten Block von der oben beschriebenen Lehrveranstaltung im WS 2016/2017. In allen drei Texten geht es um den Beginn derselben Lehrveranstaltung: 


\section{Lernjournalbeispiel 1:}

„Die erste Einheit startete mit einer Kennenlernphase, so wie es eben üblich ist. Allerdings nicht unbedingt im Rahmen einer universitären Veranstaltung - denn auf der Uni finden diese Veranstaltungen meist auf einer sehr unpersönlichen Ebene statt. Nicht so bei diesem Seminar, man merkte doch gleich, dass hier auch sehr viele Teilnehmer pädagogisch vorgebildet sind, allein schon am Umgangston etc. Nachdem ich selbst Pädagogin bin, fühlte ich mich sehr wohl und war gespannt, wohin die Reise wohl geht.

Durch die angebotenen Kennenlernspiele mussten wir uns in Kleingruppen zusammenfinden und somit wurde unsere Sozialkompetenz gefordert, wir mussten diskutieren, uns absprechen, kommunizieren und verständigen. Das Kartenspiel kannte ich noch gar nicht, fand es aber in jedem Fall sehr lustig und auch vor allem sehr sinnvoll innerhalb einer Kennenlernphase.

Ebenfalls wurde 'guter Unterricht' besprochen, wie er sein muss, welche Inhalte vorkommen müssen usw. Gerade dadurch, dass wir so eine bunte Gruppe waren, fand ich die Einwürfe der anderen Kolleginnen sehr interessant, die meisten haben schon berufliche Praxis hinter sich und konnten erzählen. Ich fand den regen Austausch sehr nett und vor allem sehr spannend.

Was mich etwas gestört hat war das dezente Chaos, das in der ersten Einheit geherrscht hat. Ich kannte mich nicht wirklich aus, welche Leistungen ich denn nun erbringen muss etc. Aber ich denke, das erging nicht nur mir so. Vielleicht sind wir aber auch nur darauf konditioniert, aufgrund der anderen Seminare und Veranstaltungen, in denen immer sehr klar und deutlich formuliert wird, was der Veranstaltungsleiter möchte. Vielleicht sind wir es nicht mehr gewöhnt, innerhalb eines gewissen Rahmens selbst zu entscheiden."

\section{Lernjournalbeispiel 2 (tabellarische Form):}

\begin{tabular}{|c|c|c|}
\hline ORGANISA- & $\begin{array}{l}\text { Das Seminar begann mit einer sehr } \\
\text { angenehmen Atmosphäre, die sehr einladend } \\
\text { war. Zu Beginn wurden die Anforderungen } \\
\text { und }\end{array}$ & $\begin{array}{l}\text { Es ist immer wichtig, sich um einen positiven } \\
\text { Start zu bemühen. Ich finde, diese gute } \\
\text { Stimmung hat sehr wesentlich dazu } \\
\text { beigetragen, dass ich mich in diesem Seminar }\end{array}$ \\
\hline TORISCHES & $\begin{array}{l}\text { Rahmenbedingungen des Seminars klar } \\
\text { kommuniziert. Es dauerte etwas, bis alle } \\
\text { Teilnehmer_innen die Anforderungen } \\
\text { verstanden haben, da diese sehr offen sind. } \\
\text { Das bedeutet, die } \\
\text { Leistungsüberprüfung ist nicht fix } \\
\text { vorgegeben sondern es obliegt den } \\
\text { Teilnehmer_innen, welche } \\
\text { Beurteilungsformen sie sich aussuchen. } \\
\text { (Referat, Lernjournal, Seminararbeit,...) }\end{array}$ & $\begin{array}{l}\text { von Anfang an recht wohl gefühlt habe. Der } \\
\text { Umgang mit den anderen Kolleginnen ist } \\
\text { sehr wertschätzend. Ich denke, das kann ich } \\
\text { mir auf jeden Fall für meine Arbeit in der } \\
\text { Praxis mitnehmen. Du bist willkommen! }\end{array}$ \\
\hline $\begin{array}{l}\text { KENNENLERNEN/ } \\
\text { SPIELE }\end{array}$ & $\begin{array}{l}\text { Medias ins res starteten wir, aber nicht direkt } \\
\text { mit inhaltlichem, nein, sondern mit sehr } \\
\text { Wichtigem! - dem Kennenlernen. Wir } \\
\text { bildeten spontan Gruppen und suchten uns } \\
\text { eine Form des Kennenlernens (verschiedene } \\
\text { Spiele) aus. Ich habe eine sehr liebe Gruppe } \\
\text { erwischt, die ich vorher nicht kannte und wir } \\
\text { spielten ein Würfelspiel mit verschiedenen } \\
\text { Fragen auf den Feldern, die dann von der } \\
\text { Person beantwortet wurden. Wir hatten } \\
\text { großen Spaß und die Fragen waren von } \\
\text { Anfang an Eisbrecher. Schnell kamen wir }\end{array}$ & $\begin{array}{l}\text { Das Spiel habe ich super gefunden! Ich habe } \\
\text { zu Hause gleich eine ähnliche Version erstellt } \\
\text { und bereits letzte Woche in einer } \\
\text { Lernhilfegruppe in } \\
\text { sonderpädagogischem Zentrum ausprobiert. } \\
\text { Es hat super funktioniert! Auch wenn ich als } \\
\text { Lehrperson in dieser Gruppe mitgespielt habe, } \\
\text { um zu koordinieren und einen Rahmen zu } \\
\text { schaffen, in dem sich alle wohlfühlen. Die } \\
\text { Kinder erzählten sehr gerne von ihren } \\
\text { Gewohnheiten, Vorlieben,... und schnell } \\
\text { waren Anknüpfungspunkte und } \\
\text { Gemeinsamkeiten gefunden. Es ist ein }\end{array}$ \\
\hline
\end{tabular}




\begin{tabular}{|l|l|l|}
\hline & $\begin{array}{l}\text { ins Gespräch und das Spiel wurde schnell } \\
\text { zur }\end{array}$ & $\begin{array}{l}\text { wirklich sehr einfaches Spiel mit großer } \\
\text { Wirkung. }\end{array}$ \\
& $\begin{array}{l}\text { Nebensache. Ich hatte ein sehr positives } \\
\text { Gefühl beim Spielen mit den anderen und wir } \\
\text { tratschten weiter in der Pause und noch nach } \\
\text { dem Seminar. }\end{array}$ & $\begin{array}{l}\text { Auch das Spiel Dixit werde ich ausprobieren, } \\
\text { ich denke damit kann man sehr viel machen! }\end{array}$ \\
\hline
\end{tabular}

\section{Lernjournalbeispiel 3:}

\section{„Einstieg in die Unterrichtseinheit}

Der Einstieg in die heutige Einheit erfolgte durch das Abklären organisatorischer Fragen. Zunächst wurde die Anwesenheitsliste und damit die Aufnahme ins Proseminar geklärt, danach wurden die Anforderungen zum Erlangen einer positiven Beurteilung besprochen.

Da ich mir diese schon vorab auf dem Informationsblatt, das auf moodle bereitgestellt wurde, durchgelesen hatte, und danach etwas verunsichert war, war dies ein sehr wichtiger Punkt für mich. Wie sich herausstellte, gab es bei vielen Studierenden offene Fragen, und diese wurden ausführlich besprochen und geklärt. Zum einen war es beruhigend für mich, zu sehen, dass auch andere Teilnehmer*innen verunsichert waren, zum anderen empfand ich es als sehr angenehm, dass schon zu Beginn alles detailliert besprochen wurde und ich mir im weiteren Verlauf keine Gedanken mehr zu diesen Fragen machen musste, da ich nun das Gefühl hatte, alles verstanden zu haben.

Für meinen zukünftigen Unterricht ist diese Struktur, Organisatorisches vorab zu klären, ein sehr hilfreicher Input. Die Lernenden können sich ohne Fragen im Hinterkopf vermutlich besser auf die Lerninhalte konzentrieren.

Anschließend an den organisatorischen Teil folgte eine „Kennenlernrunde“, in der die LVLeiterin Fragen, wie zum Beispiel Wer hat schon Erfahrung mit Unterrichten? stellte und alle betroffenen Personen die Hand heben mussten. Nach einigen Fragen wurde das „Fragerecht" an die Studierenden weitergegeben.

Ich empfand es als sehr angenehm, dass es uns beim ersten Kennenlernen in der Gesamtgruppe freigestellt war, ob wir und zu Wort meldeten. Niemand musste eine Frage stellen und die Beantwortung der Fragen erfolgte nonverbal. Ich denke, dass ich grundsätzlich ein sehr offener Mensch bin, der keine großen Probleme mit dem Sprechen vor einer Gruppe hat. Dennoch habe ich mich auf der Uni schon des Öfteren dabei beobachtet, dass erste Wortmeldungen in neuen Lehrveranstaltungen durchaus Nervosität und Stress bei mir auslösen.

Ich gehe sehr stark davon aus, dass der Einstieg von der LV-Leiterin sehr bewusst geplant wurde, um uns Studierenden ein anschauliches Beispiel von ,gutem Unterricht“ zu geben. Ich könnte mir vorstellen, dass ein Einstieg, bei dem die Kursteilnehmer*innen nicht zum Sprechen gezwungen werden, sondern sich nonverbal äußern können, gerade im

Fremdsprachenunterricht als deutlich entspannter empfunden wird. Da im DaF/Z-Unterricht in einer Fremdsprache gesprochen wird, gehe ich davon aus, dass der Stresslevel bei Wortmeldungen noch deutlich höher ist. Ich könnte mir vorstellen, dass bei einer ,üblichen“ Vorstellungsrunde (sofern überhaupt ein sprachliches Niveau besteht, in dem eine solche möglich wäre) die meisten Lernenden gar nicht richtig zuhören sondern sich vielmehr auf ihren Redebeitrag vorbereiten. In diesem Zusammenhang ist es vermutlich auch sinnvoll im Allgemeinen $\mathrm{zu}$ reflektieren, inwieweit das Auffordern zum Sprechen in den ersten Unterrichtseinheiten erfolgen sollte. Ich komme aus der Elementarpädagogik, einem Bereich, in dem es ,selbstverständlich “ ist, zu respektieren, wenn einige Kinder lange Zeit nur rezipieren 
und nicht produzieren. In der Erwachsenenbildung habe ich jedoch noch nicht so viel Erfahrung. Ich engagiere mich in einem Deutschkurs für Geflüchtete, doch obwohl ich mich sowohl im Rahmen meines Studiums, als auch privat in diesem Bereich weitergebildet habe, fühle ich mich oft noch sehr unsicher. Dieser Denkanstoß führt bei mir deshalb zu einem Hinterfragen meiner Unterrichtsgestaltung. Ich frage mich, ob ich von meinen Lernern bezüglich des Sprechens vor der Gruppe zu viel verlange. Für manche Erwachsene bedeutet es eventuell immer Stress, vor andern Menschen zu reden. Und ich habe auch bei einigen meiner Schüler beobachtet, dass es ihnen Überwindung kostet, in den ersten Stunden auf Fragen wie Wie heißt du?, Woher kommst du?, etc. zu antworten. Oft bin ich mir allerdings nicht sicher, ob es das Sprechen vor der Gruppe ist, das sie verunsichert, oder vielmehr die Angst einen „Fehler“ zu machen. Ich denke bei diesem Punkt geht es um das zentrale Thema der Lehrveranstaltung, denn Sprechen vor anderen Menschen halte ich für eine allgemeine Kompetenz. Ich bin mir etwas unsicher, inwieweit es für Erwachsenen Lerner*innen wichtig ist, sie zu ,zwingen“ über den eigenen Schatten zu springen, oder ob sich so ein Unterricht eher negativ auf das Lernen auswirkt.

Ich werde meinen Unterricht diesbezüglich zukünftig ausführlicher reflektieren. Vielleicht kann ich es schaffen, die kommunikative Kompetenz etwas zu fördern, ohne die Lerner*innen in unangenehme Situationen zu bringen. Einen Prozess zu schaffen, um auch introvertierte Lerner*innen an das Sprechen heranzuführen, ist auf jeden Fall mein Ziel.

In der Lehrveranstaltung gingen wir von der Kennenlernrunde in der Gesamtgruppe zu einer Kleingruppenaktivität über. Wir konnten zwischen verschiedenen Spielen wählen, und meine Gruppe entschied sich für ein Brettspiel, bei dem mehr oder weniger persönliche Fragen beantwortet werden mussten. Ich empfand das Spielen als sehr angenehm, da es mir auch die Möglichkeit gab, andere Teilnehmerinnen aus der Lehrveranstaltung in einer fröhlichen Atmosphäre etwas näher kennenzulernen. Mir persönlich gibt es Sicherheit, wenn mir nicht alle Personen fremd sind, und ich denke, dass das Unterrichtsklima produktiver ist, wenn die Teilnehmer*innen sich sicher und wohlfühlen.

Anschließend an die Gruppenarbeit, wurde der Einstieg in die Lehrveranstaltung thematisiert und kurz reflektiert. Wir besprachen, dass auf diese Weise die soziale Kompetenz gefördert wird, die Arbeitsfähigkeit der Gruppes erhöht wird und dass es zentral ist, den Lerner*innen zu ermöglichen, Arbeitskontakte herzustellen.

Für mich war es sehr interessant, selbst zu erleben, wie gut so ein Einstieg in eine Lehrveranstaltung funktionieren kann. Der Übergang vom Non-verbalen über die Kleingruppenarbeit hin zu einer „Diskussion“ im Plenum.

Sollte ich einmal eine größere Lerner*innegruppe unterrichten, werde ich diese Vorgehensweise auf jeden Fall im Hinterkopf behalten und darauf achten, dass die Lerner*innen sich in kleinen Gruppen austauschen können, um Kontakte zu knüpfen und Sicherheit zu erlangen. Bei Lerner*innengruppen, in denen viele verschiedenen Erstsprachen gesprochen werden und Deutsch noch nicht beherrscht wird, ist beim Einstieg vermutlich viel Kreativität gefragt. Ich könnte mir vorstellen, dass hier gut mit Bildern gearbeitet werden kann.“

\section{Lernjournale als Professionalisierungsinstrument von Dozent/innen}

Wie die Beispiele zeigen, variiert die Länge, der Fokus, der Schreibstil und der Transfer zum eigenen Unterricht je nach Erfahrung, Lust und Vorlieben. Die Analyse der Beispiele überlasse ich den LeserInnen, da dies den Rahmen dieses Beitrages sprengen würde. Die professionelle 
Analyse der Lernjournale/Lerntagebücher erfolgt meistens mit induktiver Inhaltsanalyse, evtl. mit Hilfe der Software MAXQDA. MAXQDA ist eine Software zur qualitativen Datenanalyse und/oder Textanalyse. Mit MAXQDA kann man die Ergebnisse auch visuell ansprechend darstellen.

Bisher ging es vor allem um die Lernenden und ihre Entwicklungen im Lernprozess. Darüber hinaus leisten aber Lernjournale/Lerntagebücher auch zur Professionalisierung der Unterrichtenden einen Beitrag, sofern diese Selbstreflexions-Dokumente nicht nur abgehakt oder schnell überflogen werden. Uns, den Unterrichtenden, wird durch die Begegnung der studentischen Lernjournale/Lerntagebücher eine fast einmalige Chance zuteil, unsere ganze Lehrveranstaltung mit den beabsichtigten und unbeabsichtigten Zielen, unsere Reflexe und Gewohnheiten, unsere Wirkung, die Relevanz der von uns vermittelten Inhalte, die Gruppenprozesse, also quasi unsere Dozentenleistung in einem Spiegel zu betrachten. Sofern wir unseren Studierenden genügend Zeit widmen können und für ihre in den Lernjournalen/Lerntagebüchern beinhalteten Rückkopplungen offene Augen und Ohren haben, können wir uns als Unterrichtende mit Hilfe der Lernjournale/Lerntagebücher besser weiterentwickeln, als mit Hilfe der zentralen, administrativen Qualitätssicherungssystemen und Maßnahmen. 


\section{Literaturverzeichnis:}

1. Cline, Ernest (übersetzt von Hannes und Sara Riffel) 2012. Ready Player One. München: Penhaligon Verlag. ISBN-13: 978-3764530907

2. Gibbs, Graham 1988. Learning by Doing: A guide to teaching and learning methods. Further Education Unit. Oxford: Oxford Polytechnic. ISBN. 1853380717

3. Schön, Donald A. 1983. The Reflective Practitioner: How Professionals Think in Action. London: Temple Smith. ISBN-10: 9780465068784 